\title{
CFD Analysis of a Backward Facing Step Flows
}

\author{
Senan Thabet ${ }^{1}$, Thabit H. Thabit ${ }^{2 *}$ and Yaser A. Jasim ${ }^{3}$
}

1University of South Wales, Cardiff, UK

${ }^{2}$ Ninevah University, Mosul, Iraq

${ }^{3}$ Cihan University, Erbil, Iraq

\begin{abstract}
This paper introduces a lot of situations about the Computational Fluid Dynamics (CFD) analysis of backward facing step flows. The main point of this paper is incompressible flow in a backward step, measured by drivers and Seegmiller experiments.

The researchers concluded that validation parameters chosen for the study are the locations of the surface hydrostatic pressure and the reattachment compression impact downstream of the step. The surface static pressures predicted by WIND using a two-equation SST turbulence model are shown below. This figure includes a comparison of earlier versions of NPARC and WIND with experimental data. The WIND code slightly overestimates the surface pressure drop from the free flow value to about $5 \%$ of the corner value of the separation area (base pressure). This may be due to the fact that the transition point is experimentally unknown and not accurately predicted by the natural transition of the turbulence model. However, this over-forecasting predicts the previous pressure drop closer to the experimental results than the AEDC PARC, TUFF, and GASP codes using the Baldwin Lomax, KE, and Cebeci, and Smith models, respectively. The rest of the forecast for WIND pressure is in good agreement with the forecast pressure.
\end{abstract}

Keywords: CFD, Turbulence, Backward Step

\author{
* Corresponding author \\ Thabit H. Thabit
}

thabit.acc@gmail.com

Address: Collage of Electronic Engineering, Ninevah University, Mosul, Iraq

Tel: +9647711886068

Manuscript Received 26.07.2018

Revised 14.09.2018

Accepted 14.09.2018

Doi:10.30939/ijastech..447973

\section{Introduction}

At moderate Reynolds numbers, the backward staircase flow is experimentally and numerically studied. The cause is to evaluate different channel expansion ratios $(\mathrm{ER}=1.43$, 2, 2.5 and 4) and inlet flow conditions (stabilization and pulsation) to analyze the structure and stability of the flow behind the step. Electric diffusion is used to measure the wall shear rate along the experimental aqueduct. Orientation-sensitive sensors detect the near-wall extent of different flow recirculation zones (primary recirculation and secondary recirculation, roof and bottom swirl). The result of the 2D numerical simulation performed in the commercial CFD software FLUENT provides additional information on the rearrangement of global flows caused by changes in operating parameters. As the channel expansion ratio increases, the steady recirculation pattern observed in laminar flow conditions becomes more complex. The experimental and numerical data obtained show the possible re-attachment length and roof swirl size. In the transitional region, nearwall flow presents an unstable characteristic that is highly sensitive to external low-frequency disturbances. It was found that forced inlet pulsation seriously affected the overall flow structure behind the step. By applying appropriate coercion at frequencies close to the global flow instability, significant reductions in reattachment length and intensification of pulsatile backwash can be achieved.

The focus of this study is incompressible flow in a backward step, measured by drivers and Seegmiller experiments. This geometry has a step height of 1: 9 tunnel exit height ratio which helps to minimize the free-flow pressure gradient due to sudden expansion. The experimental configuration also has a 1:12 step height to tunnel width ratio to minimize the three-dimensional effect. The data from the original experimenter is listed in the file bstepdata.txt. All of the calculations given below are for the zero dispersion of the top wall and are calculated using a two-dimensional grid. 


\subsubsection{Post processing:}

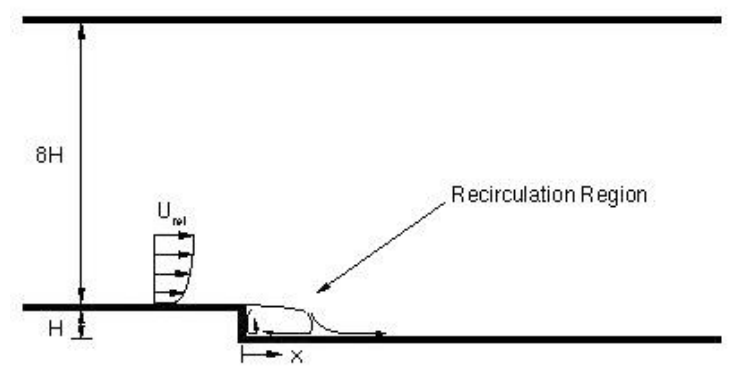

Fig. 1. Incompressible Flow in a Backward Step

\section{Problem Description}

Back stepping measurements of the WIND code versus perfect gas supersonic flow was validated. The post-phase condition provides a basic test case to evaluate the CFD code prediction of the expansion fan area between free flow and reattachment impact, the compression effect of the impact, and the relatively constant pressure area layer between reattachment and shear. WIND code with regional boundary conditions was used to determine the geometrical supersonic flow shown below and to compare with the data provided by Smith. The test case chosen was an entry traffic Mach number of 2.5 ( $\operatorname{Re}=460,000$ / in.). Under these tunnel conditions, most of the upstream plate should be laminar. The transition to turbulent conditions should occur on the background shear layer.

\subsection{The Mechanism of CFD}

Understanding and predicting fluid properties and behaviors is of particular importance in many areas, especially for design. CFD uses computations to evaluate models and solve fluid-dynamics governing equations, in general PDE. Usually, these equations are solved in three steps:

\subsubsection{Preprocessing:}

The physical domain is divided into very small area, known as the unit. The set of cells that fill the field is called a grid.

\subsubsection{Solving:}

The CFD package, known as the solver code, reads the grid and setup information, solves the equation and produces a result file containing the predicted flow characteristics (e.g., flow rate, temperature, material concentration, etc.) for each cell. Because the flow is dynamic, unstable calculations require running the solver repeatedly in many small steps. It may also take a large number of computer servers to work for hours because the grid can be very large.
The result file for useful and useful information about the resulting file helps to understand key process characteristics, report on engineering metrics (such as force or maximum temperature), and verify the quality of grids, setup parameters, and solutions.

\section{Turbulence}

Turbulence is a common phenomenon in fluid flow. Hinze (1975) presents a formal Turbulence is defined as: "a variety of irregular flow conditions the number shows random changes with time and space coordinates, and thus statistically different averages can be seen. So an absolute description is not enough Turbulence is like the chaotic fluid motion we did before, because that's possible Extract the average of important traffic, such as speed and pressure. This one what makes the turbulence model possible is the physical property.

There are usually three methods in a turbulence model: direct numerical simulation (DNS), Reynolds-average Navier-Stokes equations (RANS) and the like Recent large eddy simulation (LES).

\section{Grid}

Generate a single-area grid of $238 \times 185$ to simulate the area from $\mathrm{x} / \mathrm{H}=-105$ to +50 . The grid converges to the solid surface, making $y+=1$. Downstream of this step, 55 points are used in the recirculation zone, of which 10 are placed within $y+=30$. The grid is also near the recirculation zone to improve resolution.

This grid is provided in Plot3d (two-dimensional, multigrid, plain, and whole) and common file formats. The coordinates in both files are in feet and the height of the step $\mathrm{H}$ is $0.5 \mathrm{inch}$.

\subsection{Initial conditions}

The initial (free flow) condition is generated by WIND at startup. The Chien k-epsilon model is initialized from the existing solutions of the SST model after 35000 iterations and the turbulent viscosity.

For NPARC calculations, the Baldwin-Lomax model is used for the first 1000 iterations. At that time, the Chien k- $\varepsilon$ model was initialized from the existing turbulent viscosity.

\subsection{Boundary conditions}

Since the process is modeled using a single region, the step itself is modeled using the viscous wall stripe boundaries surrounding the hole. Downstream from this step, the $\mathrm{J} 1$ boundary is also set as a viscous wall. On the Jmax boundary, the first 12 grid points are considered as nonsticky walls in order to try and match the boundary layer profile at $\mathrm{x} / \mathrm{H}=-4$. The rest of the boundary is modeled 


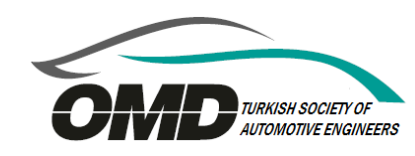

using viscous wall conditions. The I1 boundary from $\mathrm{J}=55$ to 185 is set to an arbitrary inflow, and the IMAX plane is set to a closed outflow.

\subsection{Calculation strategy}

Use WIND's time propulsion ability to make calculations to enter steady-state (asymptotic) solutions. Use local time steps for each iteration. Go ahead with time until you reach the convergence criteria. For these cases, the overall flow behavior of the solution is monitored for changes in mass flow, displacement thickness and momentum thickness.

Solving a specific problem usually involves the discretization of the first physical domain Flow occurs in an interior of a radiator such as a turbine engine or in a radiator system of a car. For very simple geometry (such as rectangular or circular), this discretization is very simple, But for more complex objects, CAD is a dilemma. Currently automatic" grid Generator "is not enough, it takes a lot of time to invest Scientist or engineer. This leads to problems in the Human Interface (HCI) and CASE Tools, as well as the basic problems of graph theory, are the result of discretization. A grid is best handled as a graph on discrete grids, the NavierStokes equations take the form of large systems. Nonlinear equations; a continuum to a discrete set of equations is a problem Combines physics and numerical analysis; for example, maintenance is important Discrete equations of mass conservation. Each node in the grid is between 3 and 20 Variables are related: pressure, three velocity components, density, temperature, In addition, physical phenomena such as capturing turbulence are also needed Part of the very nice grid in physics. Currently with 2 to 20,000 grids nodes are common, resulting in systems with up to 4000000 unknowns.

Note that the Chien k- $\varepsilon$ solution was initialized from the corresponding Menter SST solution over 35,000 iterations. Therefore, the k-epsilon model runs 55,000 iterations after initialization. When studying the convergence of the Chien $\mathrm{k}-\varepsilon$ solution, it seems that the model converges almost to 75,000 iterations. Additional iterations were performed to confirm this.

Also note that the SST solution initially ran at an outflow mass flow rate of $3.25 \mathrm{lbm} / \mathrm{sec}$ to comply with the NPARC specifications. However, the NPARC code provides only $3.20 \mathrm{lb} / \mathrm{sec}$ mass flow. In the 75,000 iterations of the SST model, the WIND boundary conditions are modified to match this actual value. After 25,000 iterations, the mass flow is almost stable at the correct value. However, the secondary surplus has not yet been fully resolved.

The WIND k-epsilon solution was initialized from SST solution to 35,000 iterations. At this point, the effluent mass flow is adjusted to the correct value.

One thing to emphasize is that a large part of these iterations are spent on the flow field to get the proper mass flow. If these conditions run again, users can expect better con- vergence (possibly up to 20,000 iterations savings).

\subsection{Convergence}

Convergence information can be obtained from the WIND output file listed above by using the result utility.

\subsection{Post-processing}

Since this study compared the NPARC and WIND results, a common postprocessor (bsteppost.f) was used. The program reads the solution from the NPARC restart file, which means that the WIND solution must be converted before it runs.

\subsection{Results comparison}

The figure (2) below shows the speed profiles for several axial positions. All profiles are normalized to the flow rate before the next step (Uref $=44.2 \mathrm{~m} / \mathrm{s}$ ). Upstream of this step, all the solutions are actually the same. However, there are significant differences within the recirculation zone. The near-indistinguishable NPARC and WIND k-epsilon solutions seem to provide the best match with the experimental data. Using the variable $\mathrm{C}$ option in the WIND k-epsilon model causes traffic to reconnect downstream, but the rest of the velocity profile cannot be predicted as well as the standard k- $\varepsilon$ model.

The WIND SST model predicts the reconnection of farthest downstream traffic.
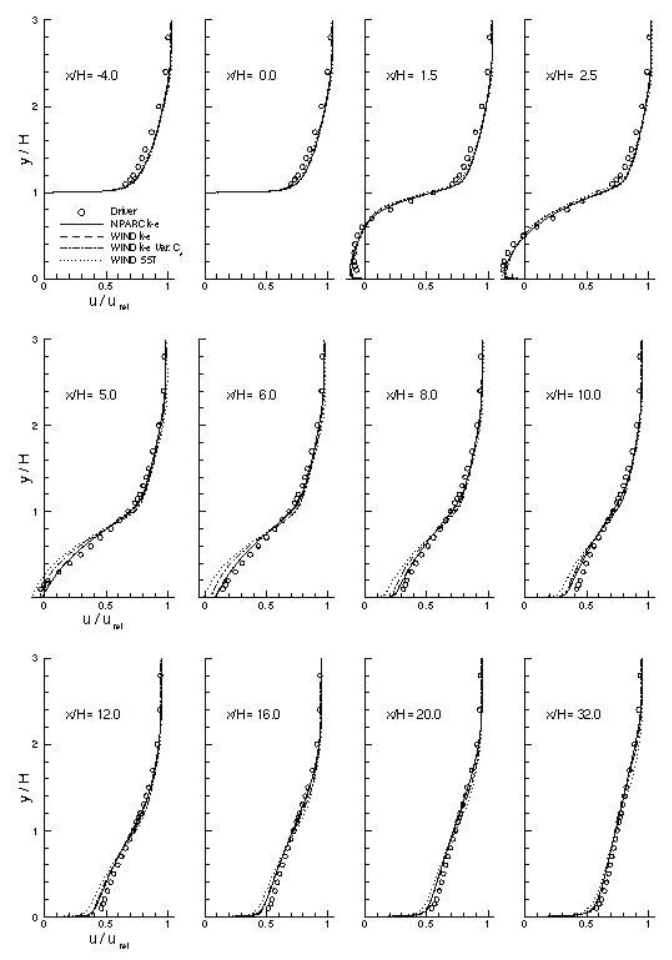

Fig. 2. Velocity Profiles at Several Axial Locations. 
in the recirculation zone.

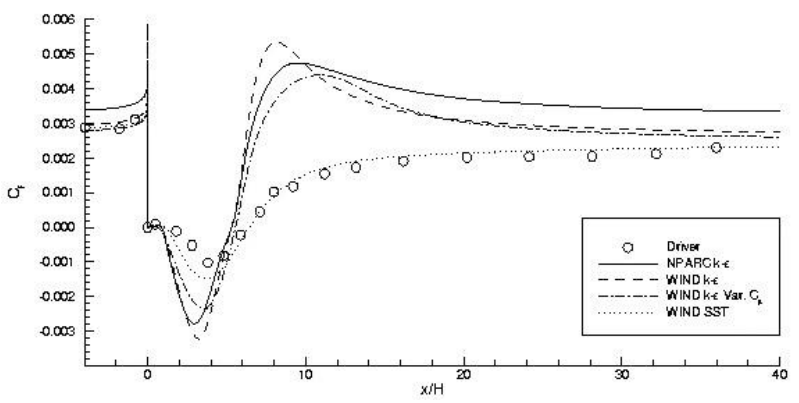

Fig. 3. Skin Friction Coefficient Downstream of Back step

In figure (3) below, these findings related to the recirculation zone are reiterated, which shows the predicted skin friction coefficient. Both the NPARC and WIND k-epsilon models predict that reattachment occurs upstream too far and show below and beyond relative to the data.

According to Avva, Smith and Singhal, this overshoot can be reduced by increasing the number of points below $y$ $+=30$. For a grid of ten points in this area, the current results are consistent with the results presented by Avva.

The variable $\mathrm{C}$ option tends to reduce turbulent viscosity within the separation area, making the flow appear more delaminated and reduces the amount of surface friction. The predicted reattachment position also shows a shift to the downstream.

Unlike the velocity profile, the WIND SST model seems to provide better agreement with surface friction data. The following table lists the predicted reattachment locations for each model.

Table 1. The Predicted Reattachment Locations

\begin{tabular}{cc}
\hline Model & $\mathrm{xr} / \mathrm{H}$ \\
NPARC k-e & 5.31 \\
WIND k-e & 5.30 \\
WIND k-e Var. C & 5.55 \\
WIND-SST & 6.43 \\
Experiment & 6.26 \\
\hline
\end{tabular}

The figure (4) below shows the distribution of turbulent kinetic energy at several axial locations. Upstream in the background, the flow is similar to that of the slab, and it can be seen that due to the k-omega model used in the near-wall region, the peak of turbulent kinetic energy is underestimated by the SST model. This difference seems to propagate downstream because the SST model is significantly lower at each axial station than the k- $\varepsilon$ model peak.

As with the velocity profile shown above, there is also a great deal of consistency between the NPARC and WIND $\mathrm{k}-\varepsilon$ solutions. One can also note that the turbulent kinetic energy is reduced due to the variable $\mathrm{C}$ option, especially in the recirculation zone. This is because the variable $\mathrm{C}$ option effectively increases the rate of turbulence dissipation with-
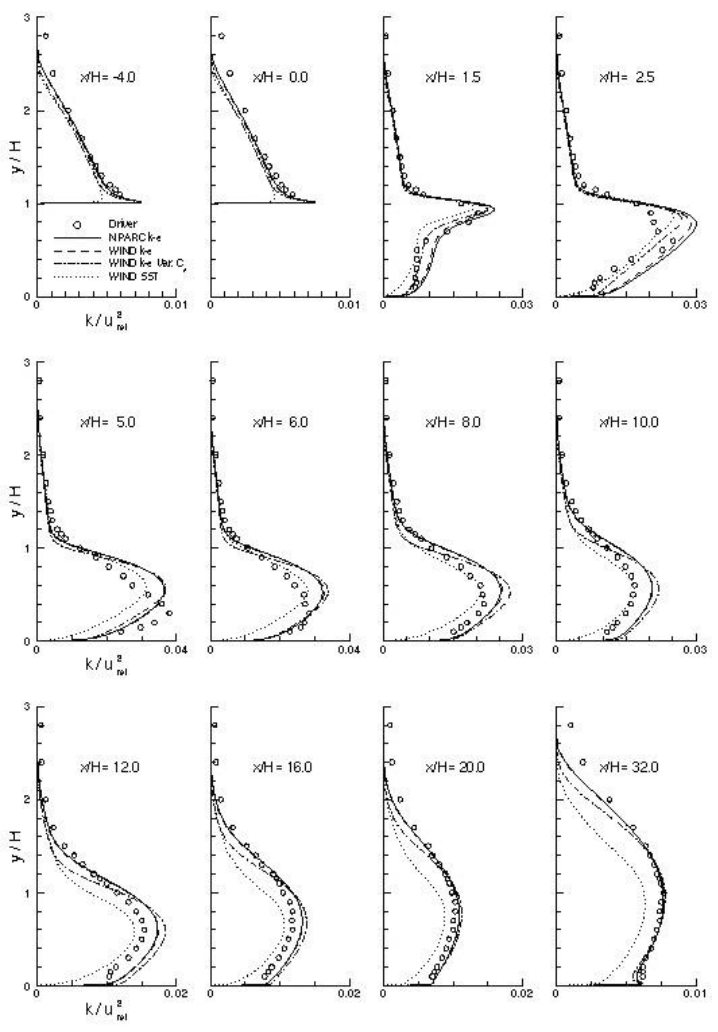

Fig. 4. Turbulent Kinetic Energy Profiles

The corresponding Reynolds stress distribution is shown below. In addition to being near $\mathrm{x} / \mathrm{H}=0$, the WIND solution predicts a rapid increase in the down-going component of velocity, which is in good agreement with the NPARC and WIND k- $\varepsilon$ solutions.

The k-epsilon model overestimates the Reynolds stress far downstream, which corresponds to the above-mentioned over-prediction of skin friction. One can also observe how variable C correction significantly reduces peak turbulent viscosity (and thus Reynolds stress) within the recirculation zone.

\section{Geometric model}

The rearward step height is 0.443 inches. The modeling domain model expands step upstream 4.0 inches, steps downstream 12 inches, steps 6.25 inches above.

\subsection{Grid}

Mesh is divided into two consecutive grid lines across the boundary of the computational domain. The upstream field is modeled with $\mathrm{I}=30 \times \mathrm{J}=40$ cells (I flow direction, $\mathrm{J}$ perpendicular to the wall). The downstream domain uses a $60 \times 79$ grid. The first point on the viscous wall corresponds to a $\mathrm{y}+$ value of about 1.0 . 


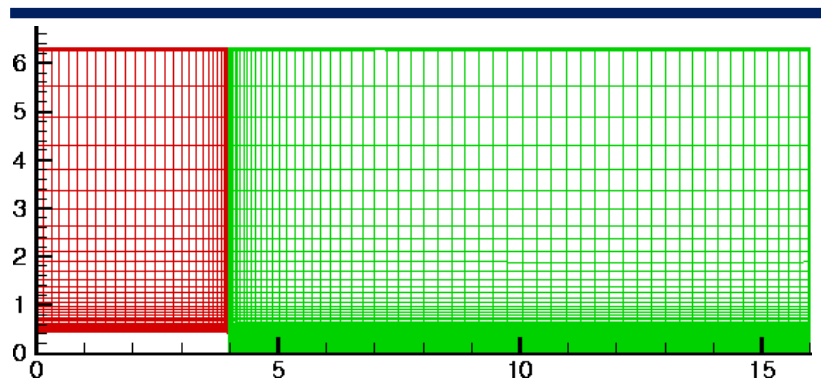

Fig. 5. The Geometric Model

\subsection{Initial and boundary conditions}

The following boundary conditions are set:

\subsubsection{Area 1:}

Left border - Freestream inflows

Right border - Coupled to grid area 2

Bottom border - sticky wall

Top border - no sticky wall

\subsubsection{Zone 2:}

Left Bound - Coupled to Grid 1, J = 40 to 79,

$\mathrm{J}=1$ to 39 sticky wall

Right Border - Freestream Outflow

Bottom border - sticky wall

Top border - no sticky wall

The initial conditions are:

\subsubsection{Area 1:}

Free flow conditions: Mach number 2.5, static $\mathrm{P}=2.227$ psi, static $\mathrm{T}=304.5$ degrees. $[\mathrm{R}$

\subsubsection{Zone 2:}

Free flow pressure and arbitrary temperature: static $\mathrm{P}=$ $2.227 \mathrm{psi}$, static $\mathrm{T}=275.4^{\circ} \mathrm{C}$. [R

Mach 0.0 the following steps (to avoid problems with the step face)

Steps above Mach 2.5

\subsection{Calculation strategy}

The computational strategy is to start flowing above the steps of Zone 1 and Zone 2 at free-flow static pressure, temperature, and Mach, while the flow following the Step 2 zone is initialized to no flow under free-flow static conditions. This initialization is intended to allow the development of step recirculation zones without causing problems to the walls after the steps. The fully implicit solver is used at local time, with a $0.40 \mathrm{CFL}$ number.

\subsection{Enter parameters and files}

WIND input file (back.dat) is:

Supersonic backward steps

Mach 2.5 Ts $=304.5$ degrees. $\mathrm{R}$ Ps $=2.227 \mathrm{psi}$

WIND Verification 10/9/97

FREESTREAM STATIC 2.5 2.227 304.5 0.0 0.0

ARBITRARY INFLOW (zero flow behind initialization region 2)

STATIC

ZONE 2

IJK_RANGE 161140110.02 .227275 .40 .00 .0

END INFLOW

Implicit boundary on

CYCLES 1000

CFL \# 0.4

Turbulent sst

End

The "ARBITRARY INFLOW" module is for initialization purposes only and will be ignored when the solution restarts, i.e. when a valid .cfl file already exists.

\subsection{Calculation}

Calculations are performed on SGI Indigo 2 running the 5.3 compiler. WIND code through the WIND script to run, and specify the default code version. The response to all filename requests (ie .dat, .cgd, .cfl, .lis) is the root name "back" (note the input file name above). Use the default solver to perform calculations that use the SST turbulence model to calculate turbulent stress. Implicit boundary conditions are used. The region 1 traffic variable converges to the L2 residual of 6.6e-06 and the turbulence variable converges to $6.4 \mathrm{e}-08$. Region 2 traffic variables converge to 2.0e-04 and turbulence variables converge to 3.2e-07.

\subsection{Post-processing}

Graphical results were obtained using FAST. Use the b4wind program to get the PLOT2D grid file and the back.cgd file to an unformatted plot $2 d$ file called back_2zone.p2d. Two PLOT2D solution files were created using the cfpost program. The first file is created by entering cfpost <cfpost_flo.inp. This produces a standard PLOT2D solution file, back_prt.2d. Create a second PLOT2D solution file by entering cfpost <cfpost_prt.inp, which creates a file, back_prt.2d, that contains the speed, static pressure (see below) normalized by free flow stagnation pressure, temperature, and density. 


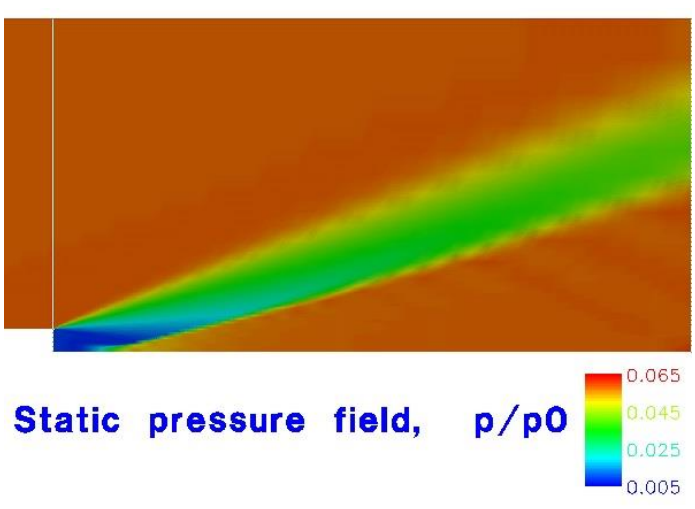

Fig. 6. Graphical Results

\section{Conclusion}

The validation parameters chosen for the study are the locations of the surface hydrostatic pressure and the reattachment compression impact downstream of the step. The surface static pressures predicted by WIND using a twoequation SST turbulence model are shown below. This figure includes a comparison of earlier versions of NPARC and WIND with experimental data. The WIND code slightly overestimates the surface pressure drop from the free flow value to about $5 \%$ of the corner value of the separation area (base pressure). This may be due to the fact that the transition point is experimentally unknown and not accurately predicted by the natural transition of the turbulence model. However, this over-forecasting predicts the previous pressure drop closer to the experimental results than the AEDC PARC, TUFF, and GASP codes using the Baldwin Lomax, KE, and Cebeci, and Smith models, respectively. The rest of the forecast for WIND pressure is in good agreement with the forecast pressure.

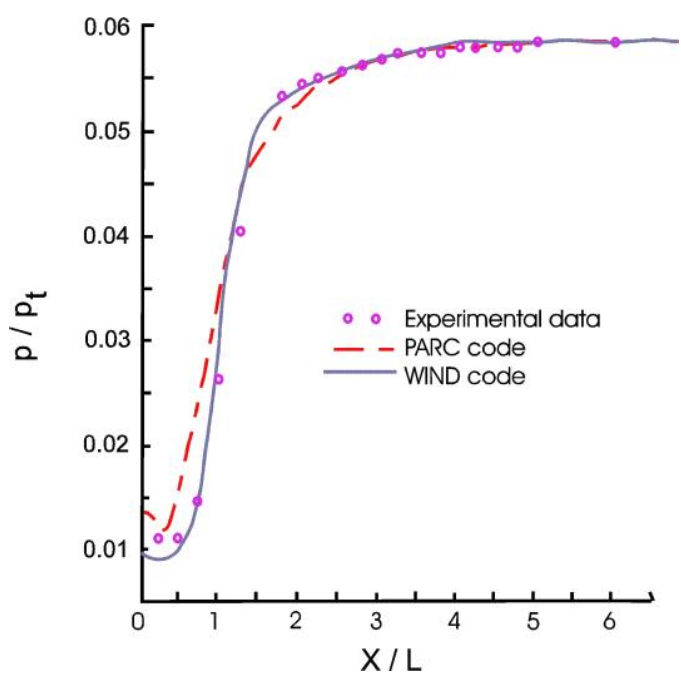

Fig. 7. The Concluded Results

\section{References}

[1] Smith, H. E. (1967). The flow field and heat transfer downstream of a rearward facing step in supersonic flow (No. ARL-67-0056). Aerospace Research Labs Wright-Patt

[2] Garrard, G. D., \& Phares, W. J. (1990). Calibration of the PARC Program for Propulsion-Type flows (No. AEDCTR-90-7). Arnold engineering development center arnold afb tn.

[3] Ebrahimi, H. B. (1995). CFD Validation and Evaluation for Reacting Flows, Part III. AIAA Paper, 735, 1995.

[4] Avva, R., \& Singhal, S. A. (1990). AIM 90-0246 Comparative Study of High and Low Reynolds Number Versions df ke Models.

[5] Driver, D. M., \& Seegmiller, H. L. (1985). Features of a reattaching turbulent shear layer in divergent

[6] Meri, A., Wengle, H., \& Schiestel, R. (2001). DNS and LES of a backward-facing step flow using 2nd-and 4thorder spatial discretization and LES of the spatial development of mixing of turbulent streams with non-equilibrium inflow conditions. In Numerical Flow Simulation II (pp. 268-287). Springer, Berlin, Heidelberg.

[7] Yoder, D. A., \& Georgiadis, N. J. (1999). Implementation and validation of the chien k-epsilon turbulence model in the wind navier-stokes code.

[8] Meri, A., \& Wengle, H. (2002). DNS and LES of turbulent backward-facing step flow using 2nd and 4th-order discretization. Fluid Mechanics and Its Applications, 65, 99114.

[9] Nallasamy, M. (1987). Turbulence models and their applications to the prediction of internal flows: a review. Computers \& Fluids, 15(2), 151-194.

[10] Speziale, C. G., \& Ngo, T. (1988). Numerical solution of turbulent flow past a backward facing step using a nonlinear K- $\varepsilon$ model. International journal of engineering science, 26(10), 1099-1112.

[11] Thangam, S., \& Hur, N. (1991). A highly-resolved numerical study of turbulent separated flow past a backwardfacing step. International journal of engineering science, 29(5), 607-615.

[12] Kim, S. W. (1988). A fine grid finite element computation of two-dimensional high Reynolds number flows. Computers \& fluids, 16(4), 429-444.

[13] Kobayashi, Y., Morinishi, Y., \& Togashi, S. (1993). Estimation of anisotropic k- $\epsilon$ model on the backward-facing step flow by LES data base. Journal of Wind Engineering and Industrial Aerodynamics, 46, 77-84.

[14] Armaly, B. F., Durst, F., Pereira, J. C. F., \& Schönung, B. (1983). Experimental and theoretical investigation of backward-facing step flow. Journal of fluid Mechanics, 127, 473-496.

[15] Thabet, S., and Thabit T.H. (2018). Computational Fluid Dynamics : Science of the Future, International Journal of Research and Engineering, 5(6) 430-433. 
[16] Thabet, S., Thabit T.H. (2018). CFD Simulation of the Air Flow around a Car Model (Ahmed Body), International Journal of Scientific and Research Publications,8(7) 517525.

[17] Thabit T.H., and Younus, Saif Q., (2018), Risk Assessment and Management in Construction Industries, International Journal of Research and Engineering, 5(2) 315-320.

[18] Thabit T.H., and Jasim, Yaser A., (2016), A Manuscript of Knowledge Representation, International Journal of Social Sciences \& Economic Environment, 1(1) 44-55. 